\title{
Can the Efficiency of a Haptic Display be Increased by Short-Time Practice in Exploration?
}

\author{
Gunnar Jansson \\ Department of Psychology \\ Uppsala University \\ Box 1225, SE-751 42 Uppsala, Sweden \\ +46183664 40 \\ gunnar-jansson@swipnet.se
}

\author{
Anna Ivås \\ Department of Psychology \\ Uppsala University \\ Box 1225, SE-751 42 Uppsala, Sweden \\ +4621145204 \\ annaivas@hotmail.com
}

\begin{abstract}
The main aim was to investigate if short-term practice in exploration with a PHANToM can improve performance. A second aim was to find out if some exploration modes are more successful than other modes. Ten participants practiced exploration of nine blocks of 24 virtual objects distributed over three days. The result was that the performance for a majority improved during this practice, but that there were large individual differences. It was suggested that one of the modes has some advantage. A main conclusion is that there is a high risk that studies of displays with users without practice underestimate their usefulness.
\end{abstract}

\section{Keywords}

Haptic display, exploration, practice, visually impaired

\section{INTRODUCTION}

An ideal computer display should present information in such a way that a user immediately, without any special practice, can pick up the information it makes available. Concerning visual and auditory displays this goal is reached in many cases. For haptic displays this requirement is much more difficult to achieve.

One explanation of this contrast between displays for the different senses is probably that the eyes and ears can explore many common displays in a way very similar to their natural ways of exploring the environment. This is not the case with the hands exploring the haptic displays presently available.

\section{Restriction to One Point of Contact at a Time between User and Virtual Object}

The exploration methods accessible for a commercially available haptic display, such as the three-degrees-offreedom versions of the PHANToM (Sensable Inc.), are restricted by the construction fact that there is only one point of contact between user and virtual scene at a time. Normal haptic exploration is usually quite different. When all fingers and both hands can be used there are many points of contact between the exploring hand and the virtual scene, and there are a number of different ways of exploring an object [9]. A six-degrees-of freedom device, such as a recently developed PHANToM, increases the available information but it is still far from the natural situation.
For the contact between the user and the virtual scene there are two standard options with a PHANToM, one with a finger put into a "thimble" and one with several fingers holding a stylus. As the number of fingers involved and ways of contact are quite different in the two cases, it may be expected that the one with more fingers would be more efficient. However, in an experiment where the two options were compared there were no significant differences, neither in proportion of correctly identified forms, nor in exploration time [6, 7]. This indicates that the critical factor is their common feature, the restriction to one point of contact at a time.

The Efficiency of Haptics in Real and Virtual Contexts Haptics is typically a sense that picks up information serially. Even if it is sometimes possible to pick up information by one grasp of an object, it is much more common to explore the object by moving it in the hand or moving the hand over it. Manipulation takes time, and there is seldom the (nearly) immediate correct identification possible with vision. This is especially apparent in virtual contexts. In an experimental study with PHANToM objects in dimensions between 10 and $100 \mathrm{~mm}$ it was found that the means of exploration times varied between 10 and $23 \mathrm{sec}$ [8]. Even if one of the forms, the sphere, could be correctly identified in $100 \%$ of the cases, other simple forms had lower identification proportions, as well as longer exploration times.

However, this result does not reflect the capacity of haptics. In an experiment, where the form of virtual and real objects in dimensions between 5 and $9 \mathrm{~mm}$ was identified, it was found that the form of real objects explored naturally were always correctly identified within a mean exploration time of $2 \sec [6,7]$. The identification of virtual objects of the same forms and with the same dimensions was much slower (means down to $25 \mathrm{sec}$ as best) and much less accurate (approaching $80 \%$ as best).

There are at least two components that may be responsible for the difference in efficiency of identification between virtual and real objects. One is the earlier mentioned difference in exploratory movements accessible; another is the availability of extended skin area at the point(s) of contact between the user's skin and the virtual object. That the latter component is important was demonstrated in experiments where only one point 
of contact and no extended skin area were available during haptic exploration of real objects [10].

\section{Changing the Display or the User?}

The difference in identification results between real and virtual objects indicates that the capacity of haptics is not the main problem. An improvement of these results has instead to be sought in factors of importance for the interaction between haptic display and user and include changes of at least one of them. In principle, changing the display in such a way that it is better adapted to haptics' way of functioning would be an excellent solution. The development of a six-degree-of-freedom PHANToM is an effort in this direction. However, the development of displays of such a complexity as those considered here is a most demanding task, from both a technical and an economic point of view. This fact is a good reason also to consider the option of changing the user.

Human beings have in many contexts demonstrated an admirable capability to adapt to new environments, including artificial ones in technical contexts, at least after long-time practice ${ }^{1}$. This adaptability has been utilized to a very large degree in the development of new technology. As an evident example, consider the development of transportation means: bikes, cars, airplanes, and moon rockets. Human beings have been able to adapt relatively well to such devices and use them successfully. However, the many accidents with many of them indicate that there are limits in the adaptation potentials of the users. User adaptation has often been relied upon as a main solution for the device-user interaction, but its limits should also be considered. This said, it may be stated that adaptation of the user may be a factor contributing to a solution, especially when adaptation of a device so complex and expensive as in the case of haptic displays.

\section{Accentuation of Haptic Exploration Problems when Vision is not Available}

When vision and haptics are used simultaneously to explore the same part of the environment haptics is to a large extent guided by vision. Vision has an immediate overview of the scene that haptics has not and can therefore guide the observer to the object to be explored and to parts of the object of special interest. When vision is not available during haptic exploration, for instance, when the exploring person is blind, haptic exploration problems are accentuated. In such situations an efficient interaction between a haptic display and its user is especially important.

\section{EXPERIMENTAL PROBLEMS}

(1) Can the Efficiency of Exploration with a Haptic Device be Increased by Short-Term Practice?

Most human skills can be improved by practice. Even if it is not possible to utilize all the biologically given

\footnotetext{
1 A discussion of the potentials of learning computer use from a cognitive point of view was provided by Mayer [11].
}

capacities of haptics when using a device such as a PHANToM, there is a high probability that the efficiency in exploration with this display will be improved with practice. However, it is not known what level of performance it is possible to reach and how much practice is needed to attain specific levels. The main aim with the experiment to be described was to investigate the effect of practice on the efficiency in using a haptic display during a rather short period.

More specifically, the main experimental problem was to study if short-term practice in exploration of objects with the stylus of a PHANToM can increase proportion of correctly identified object forms and decrease exploration time used?

\section{(2) Are there Differences in Efficiency between Ways of Holding the Stylus?}

It is known from studies in other contexts where haptic exploration is used that ways of exploring is important for efficiency, for instance, concerning tactile maps [1, 4]. It is a reasonable hypothesis that this is the case also concerning the use of haptic displays.

One aspect of exploration with a PHANToM stylus is the way of holding the stylus. Even if the activities are different there are similarities with holding a pen during writing. A pen is held in many different ways. There are a number of differences in the grip of the pencil, including number of fingers used and distance between pencil point and the tips of the fingers, as well as in the rotation of the wrist. There are also important changes during the development of children's writing [3, pp. 8794].

Informal observations indicate that users choose several different ways of holding the PHANToM stylus during exploration when no specific instruction is given. A second aim of the present experiment was to get preliminary indications of ways of holding the stylus that are successful and less successful, respectively. It may be hypothesized (1) that participants change their way during practice in order to be more efficient, and (2) that there are differences between more successful and less successful participants.

\section{METHOD}

\section{Participants}

Five men and five women, all sighted, with a mean age of 25 years ( $\mathrm{SD}=3$ years) participated. They were paid and all of them except one were university students. No participants had any experience in using a PHANToM.

\section{Haptic Display}

A PHANToM 1.5 A (Sensable Inc.) was used with the stylus option. (See www.sensable.com for details.)

\section{Virtual Objects}

The virtual objects consisted of four simple geometric forms (cube, sphere, cylinder and cone) in six different sizes (dimensions being between 5 and $15 \mathrm{~mm}$ and all three dimensions of each object having the same length). The objects were rendered with the program ENCHANTER based on GHOST ${ }^{\mathrm{TM}}$ SDK and written by 
Fänger \& König in cooperation with the first author of this paper [2].

In order to avoid problems for the participants to find the object to be explored, it was rendered in the center of a cubic room with dimensions $2 \mathrm{~mm}$ larger than those for each of the objects. At the start of each trial also the PHANToM point was located within the same room. The minimum distance between a part of the object and the surrounding room was thus minimum $1 \mathrm{~mm}$. In order to simplify for the observer to judge if the object or the inner walls of its surrounding room was touched, the object and the room were given different friction values, the former very low values and the room walls higher.

\section{Spatial arrangement}

The PHANToM was placed at the edge of a table with its arm extending in free space. The participant was sitting in front of the device with the center of the virtual objects roughly in the sagittal plane and at the height of the elbow. The stylus was grasped with the participant's preferred hand and his/her forearm was approximately horizontal.

\section{Procedure}

The participants were first informed about the functioning of the PHANTOM, the procedures of the experiment, and safety aspects. Then their eyes were covered and they were equipped with headphones providing white noise masking environmental sounds. For safety reasons they wore a standard head protective device common in industry.

The participants were instructed that their task was to identify the form of the object explored by saying the name of the form within a maximum time of $1 \mathrm{~min}$. There was no specific instruction about how to hold the stylus; the participants were only advised to hold it in a way they considered most suitable. They were told that it was important both to be accurate and to answer without unnecessary delay.

Before the experiment proper the participants were shown four real objects (dimensions $25 \mathrm{~mm}$ ) to be explored with a hand, each with one of the forms included in the experiment. This should eliminate any terminological misunderstanding. Next, they four virtual objects (dimensions $52 \mathrm{~mm}$ ) were presented to be explored with the Phantom stylus.

The objects were displayed one at a time in blocks consisting of all the 24 objects. The order was randomized differently within each block. In total nine blocks were explored by the participants during three different days, three blocks each day with a few minutes rest between the blocks. The number of objects each day was thus 72 and in total each participant explored 216 objects. The time for each daily session was about one hour. The time between the experimental days was maximum a week. At the end of each day session the participants were informed about their total result that day.

Time used for the exploration of each object, from the start of the exploration until the beginning of the response was registered, and all sessions were videotaped.

\section{RESULTS}

\section{Proportion of Correct Identifications}

The mean results for Proportion of correct identifications over the nine blocks of practice are presented in Figure 1. A three-ways ANOVA for the whole group demonstrated highly significant $(p<.001)$ effects of the factors block, size of object and form of object. However, there were large individual differences. A minority of the participants $(\mathrm{N}=3)$ had results close to chance level from start and they did not show any improvement. Their results were remarkably different from those of a majority $(\mathrm{N}=7)$ whose mean result for the ninth block was about the double of that for the first block. The most successful of the participants reached a result for the ninth block $(.88)$ that was more than five times that of a low level result for the first block (.17).

Among the forms the sphere was most easily identified, a result in agreement with that in earlier experiments [8]. The cone and the cylinder were most difficult to identify. There was a clear tendency for accuracy to increase with size of object, but the increase was not monotonous.

\section{Exploration Time}

The mean results for Exploration times over the nine blocks of practice are presented in Figure 2. A threeways ANOVA for the whole group demonstrated significant effects of the factors block $(p<.001)$, size of object $(\mathrm{p}<.001)$, and form of object $(\mathrm{p}<.01)$. However, as in the case of the Proportion correct, there were large individual differences. The same two groups could again be identified. The minority $(\mathrm{N}=3)$ performed the task in a much shorter exploration time than the other participants and their time was nearly the same during the first and the ninth block. The majority $(\mathrm{N}=7)$ decreased their exploration time during the course of the experiment. There was also a tendency to decrease the time for the blocks within each of the three days. 


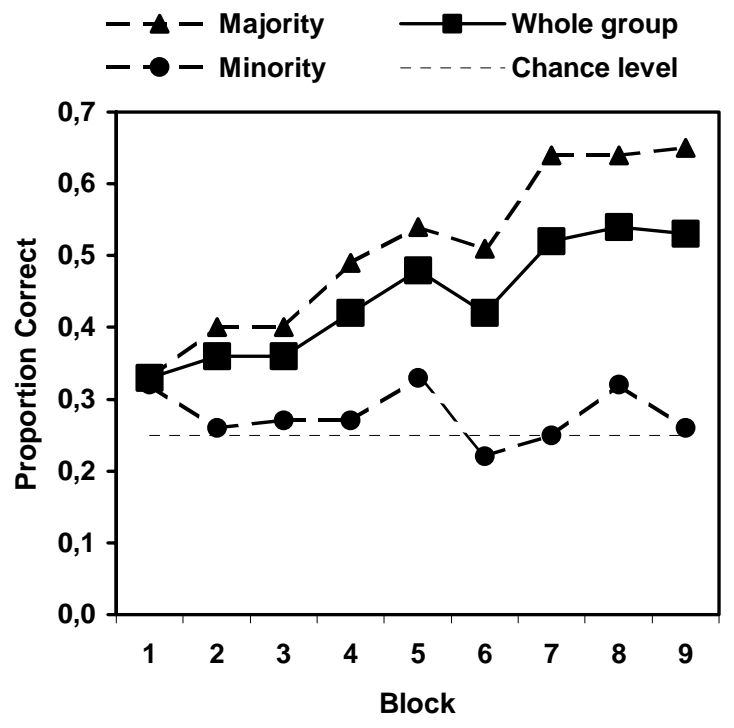

Figure 1. Means of Proportion of Correct Identifications for each block, Blocks 1-3 during Day 1, Blocks 4-6 during Day 2 and Blocks 7-9 during Day 3. Separate curves are given for Whole group, Majority, and Minority, as well as Chance level.

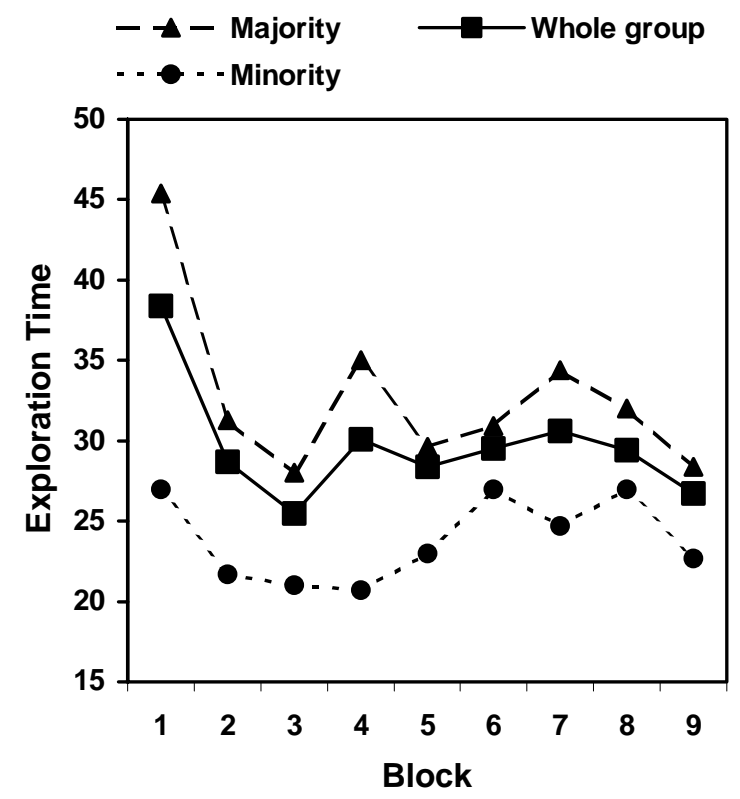

Figure 2. Means of Exploration time (sec.) for each block, Blocks 1-3 during Day 1, Blocks 4-6 during Day 2 and Blocks 7-9 during Day 3. Separate curves are given for Whole group, Majority, and Minority.

\section{Differences in Ways of Holding the Stylus}

Two main modes were used.

Nine of the participants used their right hand, one her left hand. In most cases the stylus was held closer to a horizontal plane than to a vertical plane. The stylus was grasped in mainly two ways that can be called PalmVertical and Palm-Horizontal, respectively.

The Palm-Vertical mode is similar to a precision grip $[12$, p. 86]. The stylus is in most cases held by the index finger and the middle finger opposing the thumb with the top end of the stylus protruding between the thumb and the index finger. The palm was approximately vertical (Fig. 3).

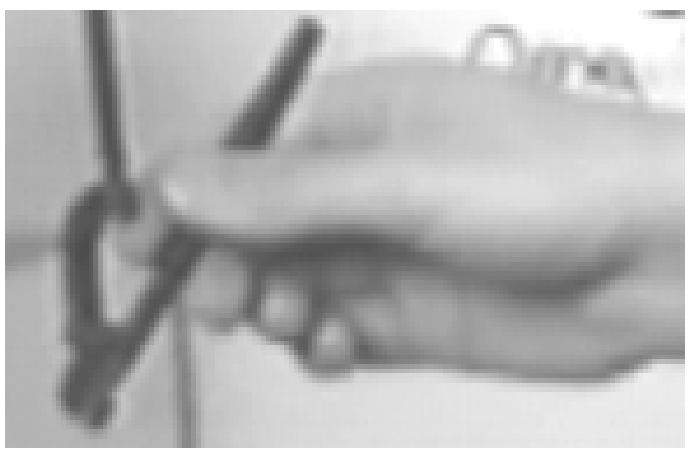

Figure 3. A Palm-Vertical mode of holding the stylus during exploration with a PHANToM stylus (photo grabbed from video).

The Palm-Horizontal mode means that the stylus is grasped from above by all fingers and the palm mainly oriented in a horizontal plane (Figs. 4 and 5).

\section{Typically, one mode was used throughout.}

Most of the participants chose one type of grasp at the start and used it through the whole experiment but three participants changed between them. Two of these changed one time (during the beginning of the third day) from Palm-Horizontal to Palm-Vertical; one participant changed a few times but used the Palm-Vertical mode during a much longer time.

\section{Advantage for the Palm-Vertical mode?}

There was no clear-cut difference in the use of the two modes between the majority and the minority mentioned above. Both modes were represented in both groups. However, it can be noted that in the successful majority the Palm-Vertical mode was used by three of seven participants throughout the whole experiment, one changed to it during the third day and one used it most of the time.

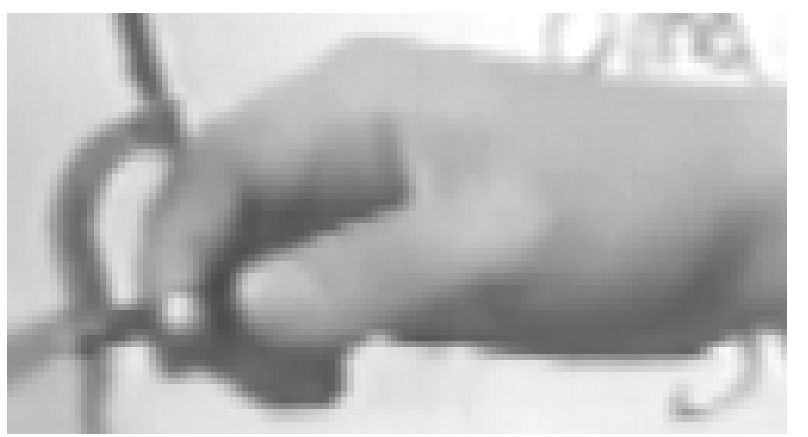

Figure 4. The Palm-Horizontal mode of grasping the PHANToM stylus during exploration (photo grabbed from video). 


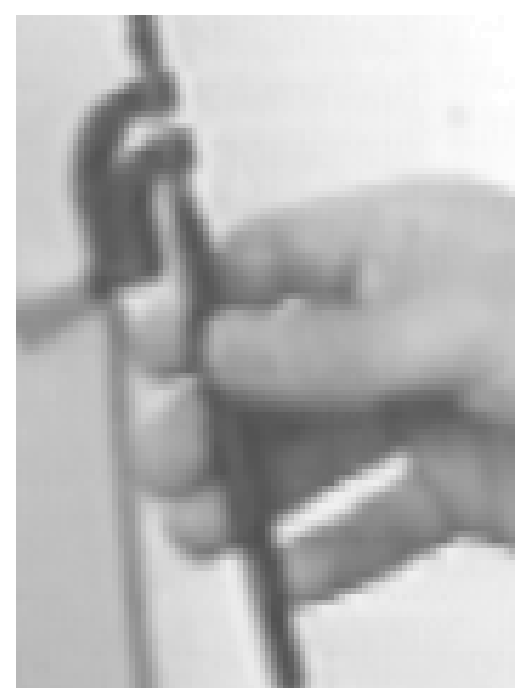

Figure 5. A temporary vertical orientation of the stylus demonstrating the grip in the Palm-Horizontal mode (photo grabbed from video).

\section{Location of Grasping on Stylus}

From the videotapes it was also studied where along the stylus it was grasped. Typically, it was held within the middle third of its length with only small variations during the experiment and between participants. No conclusion about optimal way of grasping in this respect can be drawn from the analysis.

\section{DISCUSSION}

Increased Efficiency for a Majority of the Participants The performance of the majority group of participants demonstrates that the proportion of correct identifications of virtual objects can be increased during short-time practice without any specific instruction. In the experiment the mean proportion of correct identifications for this group was approximately doubled. There is a tendency to an asymptote having been reached during the third day. No participant reached a level of $100 \%$ correct identifications, however, which is often reached with natural exploration of real objects $[6,7]$.

\section{No Improvement for a Minority of the Participants}

The factors responsible for the results of the minority group: proportions of correct identifications close to random and identifications without improvement, are uncertain. Their generally relatively short exploration times may indicate, however, that they, contrary to the instructions, gave more emphasis to speed than to accuracy $^{2}$.

\section{Efficiency of Different Ways of Holding the Stylus}

The analysis of the participants' ways of holding the stylus did not produce material for unequivocal conclusions. However, some suggestions can be found.

\footnotetext{
${ }^{2}$ One participant in the minority group grasped the stylus rather close to its top much of the time, which may have been a factor contributing to her result, as such a grasp probably decreases precision.
}

Some results indicate that the Palm-Vertical mode has some advantage. However, more research is needed to reach sure conclusions.

It should be noted, however, that it is also possible that the way of holding the stylus is not a very important parameter. Such a statement would be in line with the nodifference result obtained when the thimble and the stylus options for the PHANToM were compared [6, 7]. The dominating factor may be the one-point-of-contactat-a-time component of the haptic display.

\section{Relevance for Visually Impaired People}

One context where haptic displays may be useful is the rendering of $3 \mathrm{D}$ representations readable for visually impaired people [5]. That performance can be increased substantially by short-term practice would greatly simplify this application.

\section{CONCLUSION FOR EVALUATIONS OF HAPTIC DISPLAYS}

There is a high risk that evaluations of haptic display aiming to find an absolute level of performance and utilizing people without practice in using the device underestimate its usefulness. A few days practice in exploration may mean substantial improvements ${ }^{3}$.

\section{ACKNOWLEDGMENTS}

This study is a follow-up of projects made possible by grants from the Swedish Council for Research in the Humanities and Social Sciences and from the Swedish Transport and Communications Research Board. The authors are indebted to Lars-Erik Larsson for technical assistance.

\section{REFERENCES}

1. Berlá, E.P. Haptic perception of tangible graphic displays, in W. Schiff \& E. Foulke (eds.), Tactual Perception: A Sourcebook. Cambridge University Press, Cambridge, England, 1982, pp. 364-386.

2. Fänger, J. \& König, H. Entwicklung einer Modellierungs- und Experimentierumgebung für eine Kraftrückskopplungsgerät (Development of a form production and experiment environment for a force feedback device), Praktikumsdokumentation, Institute for Simulation and Graphics, Magdeburg University, Germany, 1998.

3. Hyldgaard, L. Spatial accuracy and temporal efficiency in children's tracing with a pencil. Ph.Dthesis, Uppsala University, Department of Psychology, Uppsala, Sweden (ISBN 91-506-14061), 2000.

4. James, G. A. Mobility maps, in W. Schiff \& E. Foulke (eds.), Tactual Perception: A Sourcebook. Cambridge University Press, Cambridge, England, 1982, pp. 334-363.

3 This problem is not so great if the aim is to find performance differences between experimental conditions. 
5. Jansson, G. Can a haptic display rendering virtual 3D objects be useful for people with visual impairment? Journal of Visual Impairment and Blindness, 93 (1999), 426-429.

6. Jansson, G. The importance of available exploration methods for the efficiency of haptic displays, in Abstracts. Symposium on Multimodal Communication, (Linköpings Universitet, Linköping, Sweden: Oct 15-16, 1999).

7. Jansson, G. \& Billberger, K. The PHANToM used without visual guidance. Proceedings of the First PHANToM Users Research Symposium (PURS99) (Deutsches Krebsforschungszentrum, Heidelberg, Germany, May 21-22, 1999). Also available at http:// mbi.dkfzheidelberg.de/purs99/proceedings/jansson.pdf.

8. Jansson, G., Billberger, K., Petrie, H., Colwell, C., Kornbrot, D., Fänger, J., König, H., Hardwick, A. \& Furner, S. Haptic virtual environments for blind people: Exploratory experiments with two devices. International Journal of Virtual Reality, 4, (1999). 10-20.

9. Lederman, S. \& Klatzky, R. L. Hand Movements: A Window into Haptic Object Recognition. Cognitive Psychology 19 (1987), 342-368.

10.Lederman, S. J. \& Klatzky, R. L Sensing and Displaying Spatially Distributed Fingertip Forces in Haptic Interfaces for Teleoperator and Virtual Environment Systems. Presence 8 (1999), 86-104.

11. Mayer, R. E. From novice to expert, in M. Helander, T. K. Landauer \& P. Prabhu (eds.), Handbook of Human-Computer Interaction (Second completely revised edition). Elsevier, Amsterdam ,1997, 781795).

12. Pheasant, S. Anthropometry, Ergonomics and the Design of Work (Sec. Ed.). Taylor \& Francis, London: 1996. 